\title{
Thermoelectric Augmenter of Heat Exchange Between the Flows of Moving Media
}

\author{
Magomadov R. A. \\ Department of Electrical Engineering and Electric Drive \\ FSBEI of HE "Grozny State Oil Technical University. \\ Acad. M.D.Millionshchikova" \\ Grozny, Russia \\ e-mail Rustmag_80@mail.ru
}

\author{
Abdulkhakimov U.I. \\ Department of Electrical Engineering and Electric Drive \\ FSBEI of HE "Grozny State Oil Technical University. \\ Acad. M.D.Millionshchikova" \\ Grozny, Russia \\ e-mail pobeda66-66@mail.ru
}

\section{Kobzarenko D.N.}

Laboratory of Information Technologies in Energy FSBUN "Institute of problems of geothermy" DSC RAS

Makhachkala, Russia e-mail kobzarenko_dm@mail.ru

\author{
Evdulov O.V. \\ Department of Theoretical and General Electrical \\ Engineering \\ FSBEI of HE "Dagestan State Technical University" \\ Makhachkala, Russia \\ e-mail ole-ole-ole@rambler.ru
}

\author{
Yusufov Sh. A. \\ Department of Theoretical and General Electrical \\ Engineering \\ FSBEI of HE "Dagestan State Technical University" \\ Makhachkala, Russia \\ e-mailyshirali@yandex.ru \\ Ragimova T.A. \\ Department of Theoretical and General Electrical \\ Engineering of FGBOU VO "Dagestan State \\ Technical University" \\ Makhachkala, Russia \\ e-mail ragimovatamila@yandex.ru
}

Ziniev S.Z

Department of Electrical Engineering and Electric Drive

FSBEI of HE "Grozny State Oil

Technical University”. Acad.

M.D.Millionshchikova

Grozny, Russia

e-mail montano_1984@mail.ru

\begin{abstract}
The paper presents the construction of thermoelectric augmenter of heat transfer. The construction uses the constrained blow of air current in the corresponding gaps by means of air moving unit with the objective to increase the coefficient of heat-exchange between thermoelectric junctions and the media moving in transport areas. The paper considers the device model built on the basis of solving the equations of thermal balance along the media flows in transport areas, thermoelectric battery surface, gaps between transport areas and battery surface for the straight flow. The theoretical research of heat transfer augmenter was carried out by means of the developed model.
\end{abstract}

Keywords-thermoelectric, heat transfer augmenter, thermoelectric battery, temperature, model, moving medium, heatexchange coefficient.

\section{INTRODUCTION}

The tasks of studying special technical devices to provide intensive heat transfer from the sources with high thermal load to heat receivers with the objective to equalize temperature levels are becoming rather urgent nowadays. These issues are especially relevant for utilizing heat energy released when performing certain technological processes in production, extracting heat from liquid refrigerating agents of nuclear reactor fuel elements, etc. Heat exchangers performing these functions are applied in aeronautical and space equipment, energy production, chemical, oil refining, food manufacturing industry, refrigeration and cryogenic engineering, heating system and hot-water supply systems, as well as conditioning system and various thermal engines. Mass and dimensions of the applied heat-exchange equipment are increasing with the growth of generating capacities and production volumes. A significant amount of alloyed and nonferrous metals is spent on their production. The reduction of mass and decrease in dimensions is a relevant problem. Heat transfer augmentation is the most perspective way to solve the stated problem. 
By now many methods of convective heat transfer augmentation have been suggested [1-5]. Such measures as turbulence promoters on a surface, rough surfaces and surfaces subjected to finning, flow twist with spiral ribs, screw systems, swirlers installed at the entrance to the channel, mixing gas bubbles to the liquid flow and mixing solid particles or liquid droplets into the gas stream, rotation or vibration of the heat exchange surface, pulsation of the heat carrier flow, the impact of electrostatic zeros on the flow, exhaustion of the flow from the boundary layer, fluid systems are utilized as applied to single-face coolants. The effectiveness of these methods is different, however, the maximum growth of heat transfer augmentation is insignificant in this case (it does not exceed 1.5-2 times) and it significantly decreases with moderate variations in temperature (tens of degrees) between media.

Boiling heat transfer augmentation provides not only heat transfer growth under the conditions of bubble and film boiling but also the augmentation of maximum heat flux with bubble boiling and minimum heat flux with film boiling as well as the increase of the corresponding dramatic temperature differences, i.e. boiling curve shift to the area of higher temperature differences and heat currents. However, the efficiency of the given methods drops as well under insignificant temperature drops.

Turbulators or ribs destroying condensate films, nonwetting coatings, and liquid stimulators for creating drop condensation, flow swirl or heat-transfer surface rotation are used under the conditions of condensation with the objective to intensify the heat transfer. Combined methods of augmentation such as combining turbulators with surface finning or with flow swirl, the application of swirlers under the conditions of suspension flow, using turbulators with low heat conductive coatings under the conditions of boiling are rather often applied. However, in this case the construction of heat exchanger, its manufacturing technique as well as reliability and durability of the equipment in case of applying turbulators become much more complicated. The use of fluid systems, porous and low temperature coatings, methods to intensify heat transfer in plate heat exchangers and finned-tube bundles has certain drawbacks linked with the coatings manufacturability, constructability of a heat exchanger, strength requirements, dirt retention, operational features, etc. All these circumstances restrict the choice of the mentioned augmentation methods in practice.

One of the most perspective directions in creating heat exchangers is the use of thermal energy converter providing the construction of efficient small-sized heat exchangers with high functionality with regards to maintaining the specified heating rate. Thus, it is possible to distinguish in this area a set of jobs which suggest allocating thermoelectric batteries equipped with ribbed surfaces on their seams between the flows of two media [6-14]. In this case the heat transfer will be augmented by means of artificial inflation of temperature difference between media due to heat emission or ingress on the seams of thermoelectric batteries.
It should be noted that the inflation of temperature difference will be concurrent with the increase of power consumed by thermal converters. Taking into account the fact that the efficiency of modern standard thermoelectric battery does not exceed $10 \%$ we can assume that such power ascension will significantly deteriorate the appliance's energy characteristics. To intensify the heat transfer under these conditions it is viable to foresee the increase of the coefficient of heat-exchange between the seams of thermal elements being the constituents of thermoelectric batteries and the moving flow in transport areas, which can be achieved by the constrained blow of air current in the corresponding gaps by means of air moving units. Taking into account that the required air moving units are fed with significantly smaller powers it is vital to conclude that practical implementation of the above mentioned technique will favour a substantial gain in thermoelectric heat transfer augmenter's energy characteristics. The present work is devoted to this research issue.

The objective of the present research is to develop and study a new construction of a thermoelectric heat transfer augmenter. Whereby, the constrained blow of air current in the corresponding gaps applied by means of air moving units will be used and the analysis of inherent heat and electrophysical processes will be conducted with the purpose to increase the coefficient of heat-exchange between the seams of thermal elements and the media moving in transport areas.

\section{METHODS AND MATERIALS}

Figure 1 present the construction of a thermoelectric heat transfer augmenter [15]. The appliance consists of a thermoelectric battery composed of identically sized elements having the same physical properties and powered by an electrical power source (which is not demonstrated in the

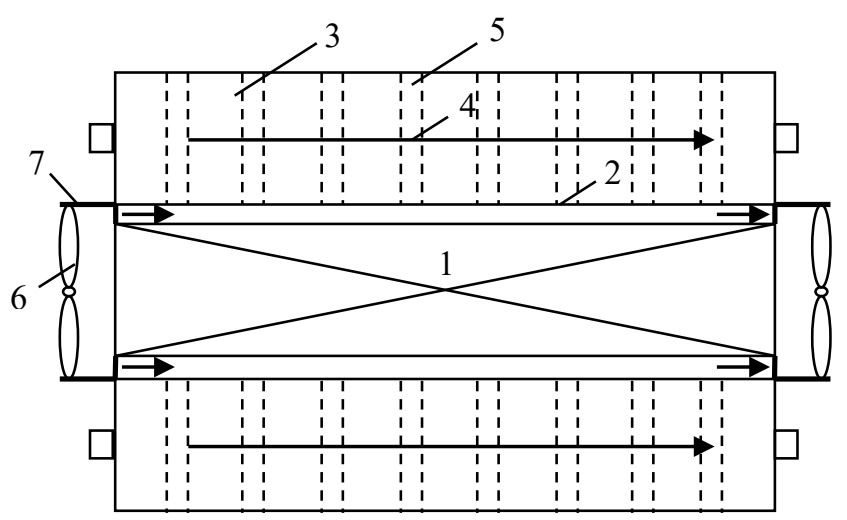

Fig. 1. Block scheme of thermoelectric heat transfer augmenter

figure). Both surfaces of the battery are at a distance (gap) from the walls of 2) transportation areas with 3) the media (4) moving in it. Through-holes (5) which form the channels for 
air flow together with gaps are made in transportation areas (3) perpendicularly to the media (4) moving direction. Air moving units (6) powered from the same source as a thermoelectric battery are installed at two ends of transportation areas (3) in the direction perpendicular to the media (4). Air moving units (6) blow the air off the gap between the walls (2) of transportation areas (3) and the surfaces of a thermoelectric battery (1). Notably, one air moving unit blows the air flow in and the second one blows it out. Thermoelectric battery (1), transportation areas (3) and air moving units (6) make a strong physical construction by means of fixing mechanisms (7).

Thermoelectric heat transfer augmenter functions in the following way: when the flow of direct current from the energy source passes through the thermoelectric battery (1), Peltier heat will be absorbed on some seams of thermal elements and will be emitted on the others. If cold seams of thermal elements will be in close proximity to the wall (2) of a transportation areas (3) with hot moving medium (4) and the hot seams of thermal elements with the wall of a transportation area with cold moving medium, the heat exchange between two media flows will be augmented by means of the present temperature drop. Herewith, the air blow off in the gaps between the walls (2) of transportation areas (3) and the surface of thermoelectric battery (1) as well as through the gaps (5) in transportation areas by means of air-to-air units (5) will enable to increase the coefficient of heat-exchange between them by means of the forced convection mode where the given coefficient value is considerably higher than with conductive heat transfer.

\section{RESULTS}

A mathematical model describing the heat and electrophysical processes in the device has been developed for the construction under consideration. The model is built on the basis of heat-balance equations concerning the media flows in transport areas, town energy balance (TEB) surfaces, gaps between transport areas and TEB surfaces [16]. The condition of straight flow is being considered.

The heat-balance equations in the gaps between transportation areas and TEB seams for the given scheme are as follows:

$$
\begin{gathered}
W^{\prime} \frac{d T_{1}}{d x}=\alpha^{\prime} L\left(T_{1 T E B}-T_{1}^{\prime}\right) \\
W^{\prime} \frac{d T_{2}}{d x}=\alpha^{\prime} L\left(T_{2 T E B}-T_{2}^{\prime}\right)
\end{gathered}
$$

where $T_{\text {ITEB, 2TEB }}$ are the temperatures of cold and hot seams correspondingly, $T_{1,2}^{\prime}$ are the temperatures of air flow in gaps and holes in transportation areas, $W^{\prime}$ is total air specific heat flown along the seams TEB (in gaps) per unit time (which is equal to the mass flow rate multiplied to the environment specific heat), $L$ is the length of transportation areas, $\alpha^{\prime}$ is the coefficient of heat-exchange between the TEB seams and an air medium in gaps.

The heat-balance equations along the media flows in transportation areas are determined by the following correlations:

$$
\begin{aligned}
& W_{1} \frac{d T_{1}}{d x}=\alpha_{1} L\left(T_{1}^{\prime}-T_{1}\right) \\
& W_{2} \frac{d T_{2}}{d x}=\alpha_{2} L\left(T_{2}^{\prime}-T_{2}\right)
\end{aligned}
$$

where $T_{1,2}$ is the temperature of the cooled and heated media, $W_{l}$ is the medium total specific heat, flown along the cold TEB seams, $W_{2}$ is the medium total specific heat, flown along the hot TEB seams, $\alpha^{\prime}$ is a coefficient of heat-exchange between the cooled air medium in a gap and a cooled air medium in a transport zone, $\alpha_{1}$ is a coefficient of heatexchange between the heated air medium in a gap and a heated air medium in a transport zone.

The heat-balance equations on TEB seams on the side of thermal elements are as follows:

$$
\begin{aligned}
& \alpha^{\prime}\left(T_{1}^{\prime}-T_{1 T E B}\right)=\bar{e} j T_{1 T E B}-\frac{1}{2} j^{2} \rho d-\frac{\lambda}{d}\left(T_{2 T E B}-T_{1 T E B}\right), \\
& \alpha^{\prime}\left(T_{2 T E B}-T_{2}^{\prime}\right)=\bar{e} j T_{2 T E B}+\frac{1}{2} j^{2} \rho d-\frac{\lambda}{d}\left(T_{2 T E B}-T_{1 T E B}\right),
\end{aligned}
$$




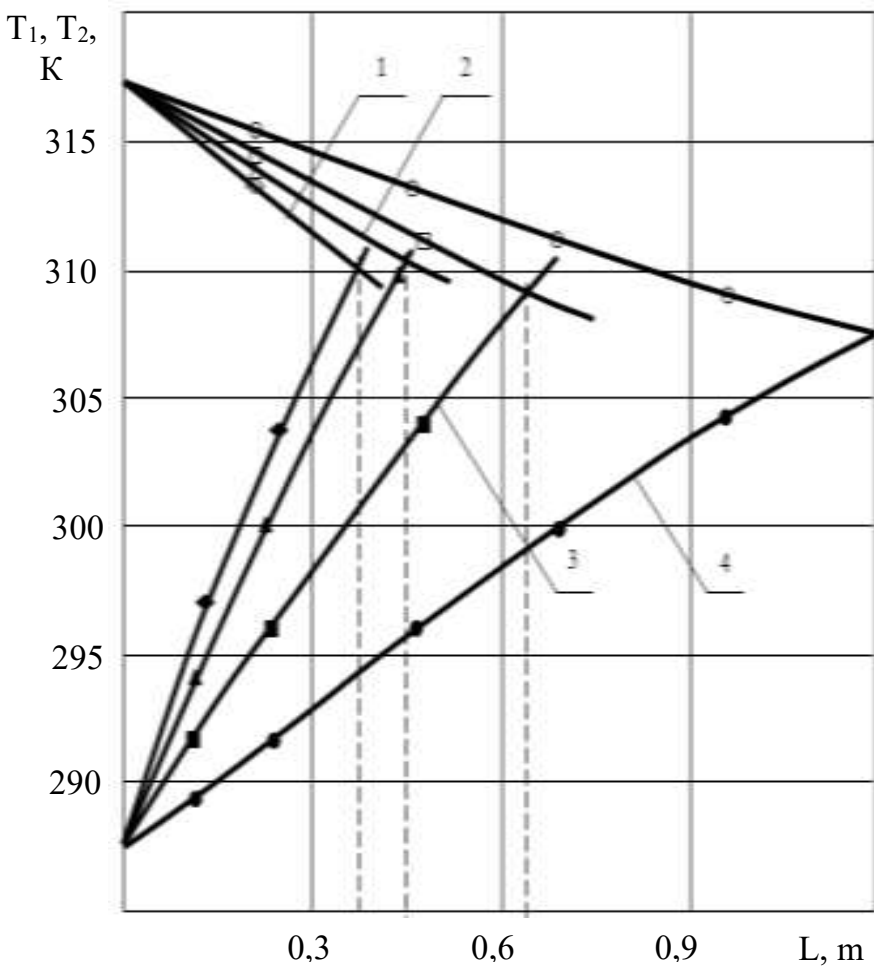

Fig. 2. Alteration of media temperatures at the output from the augmenter depending on the difference of lengths with different values $\alpha^{\prime}(1-$ $\alpha^{\prime}=90 \mathrm{~W} /\left(\mathrm{m}^{2} \cdot \mathrm{K}\right) ; 2-\alpha^{\prime}=80 \mathrm{~W} /\left(\mathrm{m}^{2} \cdot \mathrm{K}\right) ; 3-\alpha^{\prime}=70 \mathrm{~W} /\left(\mathrm{m}^{2} \cdot \mathrm{K}\right) ; \quad 4-$ $\left.\alpha^{\prime}=60 \mathrm{~W} /\left(\mathrm{m}^{2} \cdot \mathrm{K}\right)\right)$

where $\bar{e}$ is the coefficient of thermal electromotive force (EMF) of thermal elements, $j$ is the current density, $\rho$ is volume resistivity of thermoelectric branches, $\lambda$ is the distribution coefficient of thermoelectric branches thermal conductivity, $d$ is the height of thermoelectric branches.

The obtained dependences (equal to 5A) of media temperature variations at the output of the augmenter of heat exchange between the TEB seams and an air medium in a gap upon the application of TEB supply current fixed value equal to $5 \mathrm{~A}$ are demonstrated in the Figure 2. According to the obtained results we can assume that the increase of the value $\alpha^{\prime}$ enables to decrease (increase) their temperature at the output of heat exchanger under the conditions of its equal length. Thus, the alteration of $\alpha^{\prime}$ by $10 \mathrm{~W} /\left(\mathrm{m}^{2} \mathrm{~K}\right)$ at an average alters the temperature of the cooled medium by $2 \mathrm{~K}$ and of the heated medium by $3 \mathrm{~K}$.

Figure 3 shows the graph of TEB critical lengths behaviour depending on the value $\alpha^{\prime}$, i.e. the lengths whose liquids temperatures are equal to the output.

According to the research findings it is possible to conclude that the larger the temperature difference of the heat carriers at the input of the device is, the larger the TEB length required for retaining the augmentation mode is.

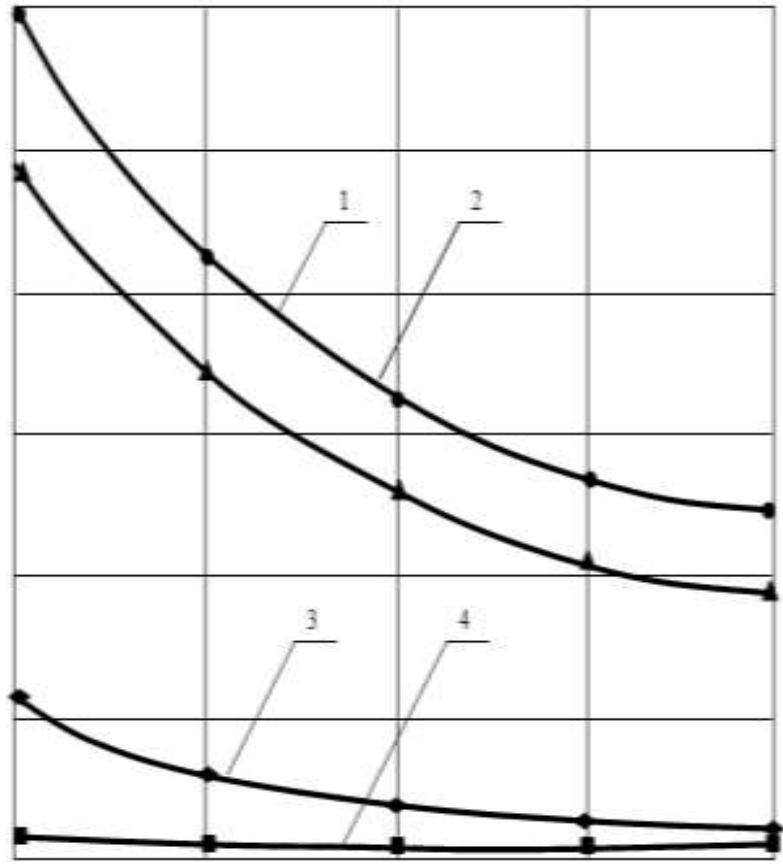

Fig. 3. Dependence of critical lengths TEB in the mode of augmentation on the fill capacity factor with different values of temperature drop at the input to the heat transfer augmenter $(1-\Delta T=40 \mathrm{~K}$;

$2-\Delta T=30$ $\mathrm{K} ; 3-\Delta T=10 \mathrm{~K} ; 4-\Delta T=5 \mathrm{~K} ; I=5 \mathrm{~A}$

The graphs have the monotonely decreasing character depending on the coefficient of heat-exchange between TEB seams and air medium in a gap. The larger the difference between the media temperatures at the output is, the more dramatically the functions $L=L\left(\alpha^{\prime}\right)$ decrease under the conditions of constant supply current $I=5 \mathrm{~A}$.

\section{CONCLUSION}

1. The construction of the thermoelectric heat transfer augmenter characterized by the application of the constrained blow of air current in the corresponding gaps applied by means of air moving units with the purpose to increase the coefficient of heat-exchange between the seams of thermal elements and the media moving in transport areas.

2. The model of the thermoelectric heat transfer augmenter has been built on the basis of heat-balance equations concerning the media flows in transport areas, TEB surfaces, gaps between transportation areas and TEB surfaces for the conditions of straight flow.

3. It has been stated that the increase of the value of the coefficient of heat-exchange between TEB seams enables to decrease (increase) their temperature at the output of heat exchanger under the conditions of its equal length. Herewith, the alteration of $\alpha^{\prime}$ by $10 \mathrm{~W} /\left(\mathrm{m}^{2} \mathrm{~K}\right)$ at an 
average changes the temperature of the cooled medium by $2 \mathrm{~K}$ and of the heated medium by $3 \mathrm{~K}$.

4. It has been stated that the increase of temperature differences of media temperatures favours the more dramatic decrease of the functions of dependences of critical TEB lengths on the coefficient of heat-exchange between the Seams TEB and air medium in a gap under the conditions of constant supply current.

\section{References}

[1] G.A. Dreitser, I.E. Lobanov, Extreme intensification of heat transfer in pipes due to artificial flow turbulization, IJF, 2003, Vol. 76, №1, pp. 4651.

[2] G.A. Dreitser, S.A. Isaev, I.E. Lobanov, Calculation of convective heat exchange in pipes with periodic protrusions, roblems of hydrodynamics and heat transfer in power plants, M .: Izd. MEI, 2003, Vol. 1, pp. 57-60.

[3] I.A. Popov, H.M. Makhyanov, V.M. Gureev, Physical fundamentals and industrial application of heat transfer intensification. Kazan. Ed. House "Logos".

[4] Yu. F. Gortyshov, V. V. Olimpiev, I. A. Popov, Efficiency of industrially effective heat transfer intensifiers, News of the Russian Academy of Sciences, Energetika, No. 3, pp. 102-118, 2002.

[5] J. Steven Browna, Piotr A. Domanskib, Review of alternative cooling technologies, Department of Mechanical Engineering, Catholic University of America, National Institute of Standards and Technology, 100 Bureau Drive, Gaithersburg, MD 20899, USA, Applied Thermal Engineering, Vol. 63, pp. 33-39, 2014.

[6] T.A. Ismailov, Thermoelectric semiconductor devices and heat transfer intensifiers, $\mathrm{SPb}$.: Polytechnic, 2005.

[7] L.P. Bulat, Applied research and development in the field of thermoelectric cooling in Russia, Refrigeration equipment, №7, pp. 3437, 2009.

[8] B.E.-Sh. Malkovich, Thermoelectric modules based on bismuth telluride alloys, Reports of the XI Interstate Seminar "Thermoelectrics and Their Applications". St. Petersburg, pp. 462-468, 2008

[9] G.A. Dreitser, Modern problems of cryogenic heat transfer and its enhancement (Generalization of experimental results. Practical recommendations and different applications), Low Temperature and Cryogenic Refrigeration. Dordrecht, Boston, London, Kruger Academic Publications, 2003, pp. 201-220.

[10] Muhammad Sajid, Ibrahim Hassan, Aziz Rahman. An overview of cooling of thermoelectric devices, School of Mechanical \& Manufacturing Engineering (SMME), National University of Sciences \& Technology (NUST), Islamabad, Pakistan, Texas A\&M University at Qatar, P.O. Box 23874, Doha, Qatar. Energy. Vol. 118, pp. 1035-1043, 2017.

[11] Kazuaki Yazawaa, Ali Shakouria,Terry J. Hendricksb. Thermoelectric heat recovery from glass melt processes, Birck Nanotechnology Center, Purdue University, West Lafayette, IN 47907, USA, NASA - Jet Propulsion Laboratory, California Institute of Technology, Pasadena, CA 91109, USA. Vol. 185, pp. 598-602, 2016.

[12] T. Zhang, New thinking on modeling of thermoelectric devices, Institute of Northern Engineering, College of Engineering and Mines, University of Alaska Fairbanks, 306 Tanana Drive, Duckering Building, Fairbanks, AK 99775, USA, Renewable and Sustainable Energy Reviews. Vol. 38, pp. 903-916, 2014.

[13] Elena Otilia Virjogheb, Diana Enescua, Elena Otilia Virjogheb. A review on thermoelectric cooling parameters and performance, Department of Electronics, Telecommunications and Energy, Valahia University of Targoviste, Unirii Avenue 18-20, 130082 Targoviste, Dambovita, Romania, Department of Automatics, Informatics and Electrical Engineering, Valahia University of Targoviste, 130082 Targoviste, Dambovita, Romania.Applied Thermal Engineering. Vol. 66, 2014, pp. 15-24

[14] S.B. Riffat, Xiaoli Ma, Thermoelectrics: a review of present and potential applications, Institute of Building Technology, School of the Built Environment, The University of Nottingham, University Park,
Nottingham NG7 2RD, UK. Applied Thermal Engineering, Vol. 64, pp. 252-262, 2014.

[15] T.A. Ismailov, O.V. Evdulov, D.K. Kadirova, D.V. Evdulov, I.Sh. Mispahov, RF Patent No. 2651112, Thermoelectric intensifier of heat transfer between flows of media with different temperatures, 2018.

[16] O.V. Evdulov, D.K. Kadirova Thermoelectric Intensifier of Flow-type Heat Transfer, Thermoelectricity, 2016, № 6, pp. 82-86.

[17] T.A. Ismailov, O.V. Evdulov, M.A. Khazamova, R.A-M. Magomadov, Mathematical model of a thermoelectric system for local thermal effects on a person's hand, Thermoelectricity, № 1, pp. 77-86, 2014 